\title{
Improved Grey Wolf Optimizer for Resource Constrained Web Service Management
}

\author{
Weimin Xiao ${ }^{1,2}$, Haojiang Deng ${ }^{1}$, Zhichuan Guo ${ }^{1+}$ and Linlin $\mathrm{Hu}^{1}$ \\ ${ }^{1}$ National Network New Media Engineering Research Center, Institute of Acoustics, Chinese Academy of \\ Sciences, Beijing 100190, China \\ ${ }^{2}$ University of Chinese Academy of Sciences, Beijing 100190, China
}

\begin{abstract}
The Grey Wolf Optimizer (GWO) algorithm is a newly proposed swarm intelligence based algorithm. It is inspired by the social hierarchy and hunting behavior of grey wolves. However, in GWO different leader hierarchy of grey wolves are considered making contributions to the search results equally. This paper proposes an Improved Grey Wolf Optimizer (IGWO) algorithm for resource constrained web service management by adding weighted position factor to diverse hierarchy of grey wolves. IGWO is tested with standard dataset of PSPLIB to evaluate the ability of web service management in resource constrained system. The results prove that the IGWO algorithm is competitive compared to well-known meta-heuristics.
\end{abstract}

Keywords: Improved Grey Wolf Optimizer, Resource Constrained, Web Service Management

\section{Introduction}

Resource constrained web service management[1][2] is one of the application scenarios in resource constrained project scheduling problem (RCPSP). RCPSP is a general scheduling problem containing limited resources, tasks of resource requests and durations. Since RCPSP is a NP-hard problem, meta-heuristics have been proposed to search for optima in efficient ways[3][4][5][6][7][8][9][10][11]. Some of the most popular meta-heuristics are Genetic Algorithm (GA)[6], Particle Swarm Optimization (PSO)[3][7][8][9], Ant Colony Optimization (ACO)[4], Simulated Annealing (SA)[10], and Cuckoo Search (CS)[11]. These algorithms are benchmarked with the standard dataset of the project scheduling problem library (PSPLIB)[12].

Meta-heuristic algorithms are becoming more and more popular for solving scientific and engineering problems. The optimization refers to the process of obtaining optimal solutions and can be found in all study fields. These population-based stochastic optimization techniques perform optimization randomly, they usually start the process by generating a set of random solutions. Over the iterations the randomly created solutions are combined, moved, or evolved. Apart from the GA, PSO, and CS algorithm, it is worth mentioning some of the other popular algorithms including Dragonfly Algorithm (DA)[13], the newly proposed Ant Lion Optimizer (ALO)[14], and Grey Wolf Optimizer (GWO)[15].

The grey wolf optimizer is one of the newly proposed meta-heuristic algorithms. This paper proposes an Improved Grey Wolf Optimizer (IGWO) algorithm for resource constrained web service management with weighted position factor to emphasize the importance of different social hierarchy of grey wolves, thus improving the optimization performance when searching for the optimum solution.

The rest of the paper is structured as follows. Section 2 describes the resource constrained web service management. Section 3 introduces the GWO algorithm. Section 4 describes the proposed IGWO algorithm. Section 5 presents the experiments and results of IGWO with PSPLIB standard datasets to simulate resource constrained web service management problem. Finally, the summary of the study is given in Section 6.

\footnotetext{
+ Corresponding author. Tel.: +8618612062648.
}

E-mail address: guozc@dsp.ac.cn. 


\section{Resource Constrained Web Service Management}

Resource constrained web service management is a scheduling problem involving web services need to be scheduled. Resource constrained web service management problem is restricted to meet constraints and achieves a certain objective. The web service management problem studied in this paper is described as: (1) Finding the minimal web services makespan schedule is the objective; (2) The number of web services is $N+2$, and each web service $i$ has processing duration $d_{i}(i=0, \ldots, N+1)$. Web services are non-preemptive during management. The web service activity 0 and $N+1$ are pseudo activities which indicate the start and finish of schedule, respectively. (3) Web services have precedence constraint. For example, if $P_{i}$ is the set of immediate predecessors of web service $i$, web service $i$ is not allowed to start until the finish of all the immediate predecessors $P_{i}$. (4) There are renewable resources in various types with constant amount at each time or period. Let $R$ be the available amount of resource $Q$. Each web service $i$ requires resource $r_{i k}$ unit of resource $k(k=1, \ldots, q)$ per unit of web service's execution time $d_{i}$. An example of web service management problem is shown in Fig. 1 , and the parameters $\left(d_{i}, r_{i k}\right)$ of web service $i$ are illustrated.

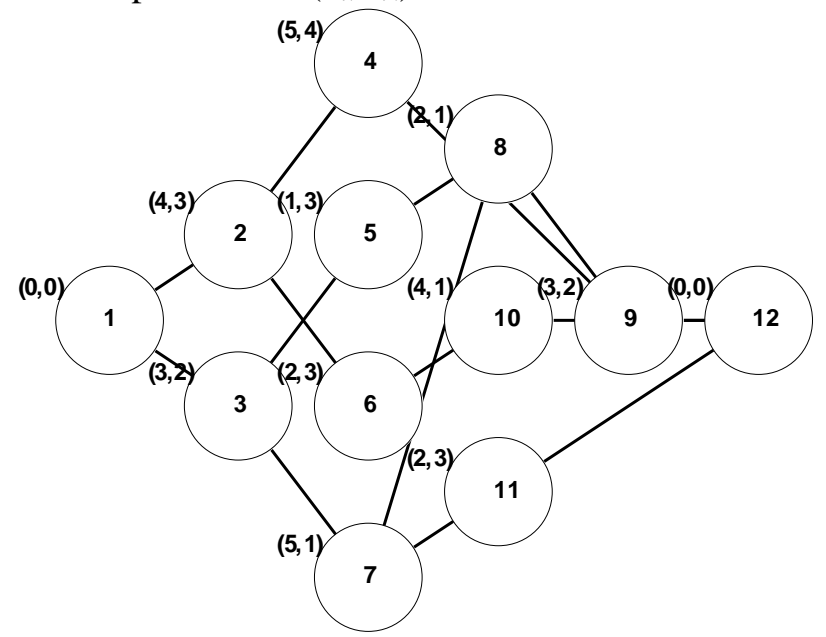

Fig. 1: Web Service Management Problem.

\section{Grey Wolf Optimizer Algorithm}

GWO algorithm is a newly proposed meta-heuristics algorithm by Mirjalili in 2014[15], and it is inspired by the social hierarchy and hunting behavior of grey wolves in nature. The grey wolves are always live in a pack and have a strict social hierarchy. The leaders who make the decision are the alpha, and the grey wolves helping alphas with decision making and other activities are called betas. The third level of grey wolves is called delta and the rest of the grey wolves are omegas. The hunting behavior of grey wolves includes tracking the prey and approaching it, encircling until the prey stops, attacking the prey.

In GWO algorithm, the social hierarchy and hunting behavior of grey wolves are mathematically modeled and simulated to perform optimization. The alpha $(\alpha)$ is assumed to be the best solution, beta $(\beta)$ and delta $(\delta)$ are considered to be the second and third optimal solutions during the optimization respectively. Omegas $(\omega)$ are the rest of optional solutions.

Firstly in the optimization, grey wolves tracking and encircle the prey. To mathematically simulate the tracking and encircling behavior, the following formulas are presented:

$$
\begin{aligned}
& \vec{D}=\left|\vec{C} \cdot \vec{X}_{p}(t)-\vec{X}(t)\right| \\
& \vec{X}(t+1)=\left|\vec{X}_{p}(t)-\vec{A} \cdot \vec{D}\right|
\end{aligned}
$$

where $\vec{X}_{p}$ is the position vector of the prey, the vector of the grey wolf is represented by $\vec{X}, \mathrm{t}$ indicates the current iteration. $\vec{A}$ and $\vec{C}$ are coefficient vectors and calculated as follows:

$$
\begin{aligned}
& \vec{A}=2 a \cdot \vec{r}_{1}-a \\
& \vec{C}=2 \cdot \vec{r}_{2}
\end{aligned}
$$

where $\vec{r}_{1}$ and $\vec{r}_{2}$ are vectors generated randomly in $[0,1]$. a represents the convergence factor linearly decreased from 2 to 0 over the iterations and is calculated as follows: 


$$
a=2-t \cdot 2 / t_{\max }
$$

where $t_{\max }$ is the total number of iteration and $\mathrm{t}$ represents the current iteration.

When attacking towards the prey, the alpha leads other wolves with beta and delta helping occasionally. During the iteration over the optimization, other search agents are obliged to update their positions according to the best three solutions obtained so far. The formula to update positions are calculated as follow:

$$
\vec{X}(t+1)=\left(\vec{X}_{1}+\vec{X}_{2}+\vec{X}_{3}\right) / 3
$$

where $\vec{X}_{1}, \vec{X}_{2}, \vec{X}_{3}$ are calculated respectively as follows:

$$
\vec{X}_{1}=\vec{X}_{\alpha}-\vec{A}_{1} \cdot\left(\vec{D}_{\alpha}\right), \vec{X}_{2}=\vec{X}_{\beta}-\vec{A}_{2} \cdot\left(\vec{D}_{\beta}\right), \vec{X}_{3}=\vec{X}_{\delta}-\vec{A}_{3} \cdot\left(\vec{D}_{\delta}\right)
$$

where $\vec{X}_{\alpha}, \vec{X}_{\beta}, \vec{X}_{\delta}$ represent the best three solutions at a certain iteration t, and $\vec{D}_{\alpha}, \vec{D}_{\beta}, \vec{D}_{\delta}$ are calculated respectively as follows:

$$
\vec{D}_{\alpha}=\left|\vec{C}_{1} \cdot \vec{X}_{\alpha}-\vec{X}\right|, \vec{D}_{\beta}=\left|\vec{C}_{2} \cdot \vec{X}_{\beta}-\vec{X}\right|, \vec{D}_{\delta}=\left|\vec{C}_{3} \cdot \vec{X}_{\delta}-\vec{X}\right|
$$

\section{Improved Grey Wolf Optimizer Algorithm (IGWO)}

The improvement of proposed IGWO algorithm is discussed in this session. A weighted position factor is presented to strengthen the diverse social hierarchy of grey wolves.

According to the design principle of GWO, alpha, beta, and delta have better knowledge of the potential position of the optimum, and other search agents should update their positions according to these three best candidate solutions at each iteration. It is noted in Eq. 3.6 the best three candidate solutions have the same weight when updating other search agents' positions. However, consider the social hierarchy of grey wolves, alpha is the fittest solution and knows the best of the potential optimum, with beta and delta in second and third places. So it is more logical to strength the impact of alpha and beta. A nonlinear weight factor of position with exponential function is proposed to enhance the difference between leader hierarchies of grey wolves. The weight factor of alpha, beta, and delta are represented by $w_{1}, w_{2}, w_{3}$ and calculated as follow:

$$
w_{1}=3-\exp \left(-30 \cdot t / t_{\max }\right)^{3}, w_{2}=2-\exp \left(-20 \cdot t / t_{\max }\right)^{3}, w_{3}=1-\exp \left(-10 \cdot t / t_{\max }\right)^{3}(3.9)
$$

where $t_{\max }$ is the total number of iteration and t represents the current iteration.

The positions of other search agents are calculated as follow:

$$
\vec{X}(t+1)=\left(w_{1} \cdot \vec{X}_{1}+w_{2} \cdot \vec{X}_{2}+w_{3} \cdot \vec{X}_{3}\right) /\left(w_{1}+w_{2}+w_{3}\right)
$$

The pseudo code of IGWO algorithm is presented in Fig. 2.

Initialize the grey wolf population $X_{i}(i=1,2, \ldots, n), a, A$, and $C$

Calculate the fitness of each search agent

$X_{\alpha}, X_{\beta}$, and $X_{\delta}$ be the best three search agents

while $(t<$ Max number of iterations)

for each search agent

Update position factor $w_{1}, w_{2}, w_{3}$

Update the position of the current search agent with weighted factor

end for

Update a, A, and $C$

Calculate the fitness of all search agents

Update $X_{\alpha}, X_{\beta}$, and $X_{\delta}$

$t=t+1$

end while

return $X_{\alpha}$

Fig. 2. Pseudo code of GWO algorithm 


\section{Experimental Results and Comparisons}

In this section, in order to investigate the IGWO for resource constrained web service management problem, a typical project example shown in Fig. 1 is analyzed, and PSPLIB datasets are used as benchmark. Fig. 3 shows the solutions and resource utilizations using 4 Algorithms (GA, PSO, GWO, and IGWO) to solve the example shown in Fig. 1. The results show that IGWO can perform scheduling better than other meta-heuristic methods. The duration time of IGWO is shorter than GA and the same as GWO and PSO.

For comparing the performances of IGWO to other popular meta-heuristics on resource constrained web service management problem, the instances in the well-known PSPLIB are used as benchmark and simulated. The standard dataset of PSPLIB contains subset J30, J60 and J120, which include 30, 60 and 120 activities respectively. In PSPLIB benchmark, the number of instances for J30 and J60 are 480, respectively, and that for J120 is 600 . Hence, there are total of 1560 instances. For each instance, IGWO and comparing algorithms were run independently with 25 search agents over 400 iterations. Statistical results (optimal found and average deviation) are collected and reported in Table 1. IGWO was compared with GWO, PSO and GA.

For all dataset, IGWO performs best comparing to other algorithms in optimal found and average deviation. The results show that the IGWO is better than other algorithms for solving resource constrained web service management problem with different scales.
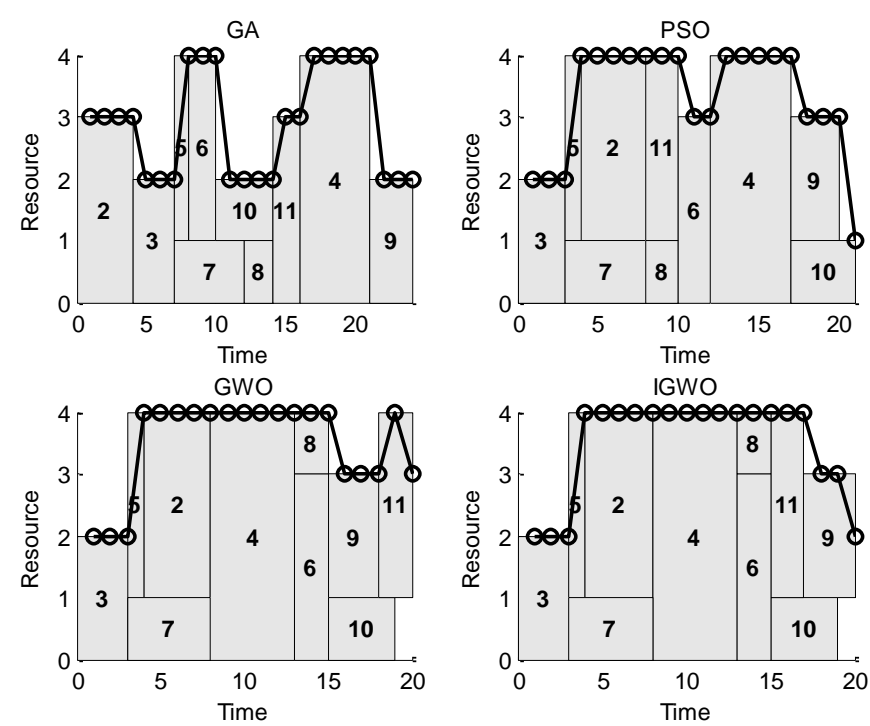

Fig. 3: Solutions and Resource Utilizations by 4 Algorithms (GA, PSO, GWO, and IGWO).

Table 1: Results of RCPSP standard dataset.

\begin{tabular}{|c|c|c|c|c|}
\hline Dataset & Algorithm & Total & Optimal Found & Average Deviation (\%) \\
\hline \multirow[t]{4}{*}{$\mathrm{J} 30$} & IGWO & \multirow[t]{4}{*}{480} & 351 & 0.531 \\
\hline & GWO & & 343 & 0.5954 \\
\hline & PSO & & 347 & 0.6001 \\
\hline & GA & & 340 & 2.6026 \\
\hline \multirow[t]{4}{*}{ J60 } & IGWO & \multirow[t]{4}{*}{480} & 214 & 11.8823 \\
\hline & GWO & & 211 & 11.8845 \\
\hline & PSO & & 209 & 12.9108 \\
\hline & GA & & 206 & 11.9205 \\
\hline \multirow[t]{4}{*}{ J120 } & IGWO & \multirow[t]{4}{*}{600} & 127 & 33.337 \\
\hline & GWO & & 116 & 34.446 \\
\hline & PSO & & 122 & 36.5 \\
\hline & GA & & 119 & 39.615 \\
\hline
\end{tabular}

\section{Conclusion}

This study presented an improved grey wolf optimization algorithm (IGWO) for solving resource constrained web service management, based on GWO algorithm. The proposed improvement is weighted position factor when updating grey wolves' position during iteration, considering the diversity of leader 
hierarchy. A typical project example is analyzed, and PSPLIB datasets are used as benchmark and simulation. By analyzing the results, IGWO was found to be competitive with other meta-heuristic algorithms.

\section{Acknowledgements}

The authors wish to thank the reviewers for their valuable comments and suggestions about improving the presentation and structure of this paper. This work was supported in part by the Special Fund for National High-tech R\&D Program (863 Program) under Grant NO. 2015AA015802 and Strategic Pilot Technology Chinese Academy of Sciences under Grant NO. XDA06040501. Zhichuan Guo is the corresponding author.

\section{References}

[1] Y. Liu. A Process Modeling-Based Approach for Web Service Management. 2009 IEEE International Conference on Web Services, Los Angeles, CA, 2009, pp. 928-935.

[2] X. Zhu, and B. Wang. Web service management based on Hadoop. ICSSSM11, Tianjin, 2011, pp. 1-6.

[3] Jia, Qiong, and Y. Seo. An improved particle swarm optimization for the resource-constrained project scheduling problem. The International Journal of Advanced Manufacturing Technology, 67.9. 2013:2627-2638.

[4] J. Yan, C. Zhao and H. Dong. Resource constrained project scheduling problem based on improved Ant Colony algorithm. The 26th Chinese Control and Decision Conference (2014 CCDC), Changsha, 2014, pp. 1560-1563.

[5] K. S. Hindi, Hongbo Yang and K. Fleszar. An evolutionary algorithm for resource-constrained project scheduling. in IEEE Transactions on Evolutionary Computation, vol. 6, no. 5, pp. 512-518, Oct 2002.

[6] H. Zhang, H. Xu and W. Peng, "A Genetic Algorithm for Solving RCPSP," 2008 International Symposium on Computer Science and Computational Technology, Shanghai, 2008, pp. 246-249.

[7] Joy J, Rajeev S, Narayanan V. Particle Swarm Optimization for Resource Constrained-project Scheduling Problem with Varying Resource Levels. Procedia Technology, 2016 Dec 31;25:948-54.

[8] Chen, Ruey Maw. Particle swarm optimization with justification and designed mechanisms for resourceconstrained project scheduling problem. Expert Systems with Applications, 38.6, 2011:7102-7111.

[9] Meng, Chunqing. "A New Particle Swarm Optimization with Multi-speed Updating Formulas." Journal of Network New Media, 2013.

[10] P. P. Das and S. Acharyya. Simulated annealing variants for solving resource Constrained Project Scheduling Problem: A comparative study. 14th International Conference on Computer and Information Technology (ICCIT 2011), Dhaka, 2011, pp. 469-474.

[11] Y. Jin, H. Nie, P. Duan and H. Yang. An improved cuckoo search algorithm for resource-constrained project scheduling problem with generalized precedence relations. 2016 12th World Congress on Intelligent Control and Automation (WCICA), Guilin, 2016, pp. 1086-1090.

[12] Rainer K, Arno S. PSPLIB-A project scheduling problem library: OR Software-ORSEP Operations Research Software Exchange Program. European Journal of Operational Research, 1997;96(1):205-16.

[13] Mirjalili, Seyedali. Dragonfly algorithm: a new meta-heuristic optimization technique for solving single-objective, discrete, and multi-objective problems. Neural Computing and Applications, 27.4, 2016:1053-1073.

[14] Mirjalili, Seyedali. The Ant Lion Optimizer. Advances in Engineering Software, 83.C, 2015:80-98.

[15] Mirjalili, Seyedali, S. M. Mirjalili, and A. Lewis. "Grey Wolf Optimizer." Advances in Engineering Software, 69.3, 2014:46-61. 